|| ISSN(online): 2589-8698 || ISSN(print): 2589-868X ||

International Journal of Medical and Biomedical Studies

Available Online at www.ijmbs.info

NLM (National Library of Medicine ID: 101738825)

Index Copernicus Value 2019: 79.34

Original Research Article

Volume 5, Issue 9; September: 2021; Page No. 21-23

\title{
FREQUENCY OF BACTEREMIA IN BURN PATIENTS IN RELATION TO THROMBOCYTOPENIA
}

\author{
Dr. Vinod Bhavi ${ }^{1}$, Dr. Jas Karan Singh ${ }^{1}$, Dr. Gurpreet Singh ${ }^{1}$, Dr. Deepak Meena ${ }^{1}$ \\ ${ }^{1}$ Department of Surgery, Guru Gobind Singh Medical College \& Hospital, Faridkot, Punjab
}

Article Info: Received 09 July 2021; Accepted 29 August 2021

DOI: https://doi.org/10.32553/ijmbs.v5i9.2176

Corresponding author: Dr. Jas Karan Singh

Conflict of interest: No conflict of interest.

\begin{abstract}
Burn patients are obviously at high risk for infections due to the immune compromising effects of burn injury. E.coli is an important life-threatening pathogen in burn units [1]. The aim of this study was to determine bacteremia in the surgical and plastic surgery units of Guru Gobind Singh Medical College and Hospital, Faridkot.25 burn patients were chosen at random study staph aureus (6) was the dominant organism followed by E. Coli (4) in blood culture,. Eight (32\%) of the total patients died and $68 \%$ survived.
\end{abstract}

Keywords: Burn, Bacteremia, Thrombocytopenia

\section{Introduction}

Patients who suffer severe burns are at higher risk for local and systemic infections. Bacteremia is an invasion of the bloodstream by bacteria. Bacteremia develops as a result of damage to the external (skin) or the internal (respiratory tract, digestive tract) barriers of the body[2].Thrombocytopenia is almost universal in bacterial infections associated with bacteremia and is usually the result of increased platelet consumption. The reduced platelet count may be an isolated finding or may be associated with disseminated intravascular coagulopathy[3].

Thrombocytopenia usually occurs early and can be an early indication of bacteremia in burn patients [4-7].According to M. Kurtoulu (astudy of 54 severely burned patients, mean body surface area burned(BSAB) $-60 \%$, mortality $-83 \%$ ) sepsis was the cause of death in $66 \%$ of cases [4].

\section{Study Materials and Methods}

Present study was conducted on 25 burn patients admitted in surgical and plastic surgery units of Guru Gobind Singh Medical College and Hospital, Faridkot. The study was conducted to evaluate burn sepsis with reference to platelet count as a diagnostic and prognostic indicator of sepsis.

All the patients were chosen at random. The extent of burn injury was determined by 'rule of nines' (Wallace).

A quick brief history regarding mode of injury and general physical examination was done in every case. The patients were resuscitated with intravenous fluids and were given appropriate antibiotics and analgesics.

Fluid calculation during first 24 hours was done by using Parkland's formula $(4 \times$ body weight $\times$ percentage of burns). Maintenance fluids were given after 24 hours according to daily losses of electrolytes, water, daily caloric needs and urine output.

Blood transfusion was given whenever it was needed. Patients were also fed orally or by nasogastric tube according to the condition of the patient. Any associated disease or illness was recorded. Routine investigations like

$\mathrm{Hb}$

BT

CT

TLC

DLC

Urine-Complete Examination

FBS

Blood urea

Serum Creatinine

Serum Electrolytes were carried out

All the patients were monitored daily regarding general condition, temperature; pulse rate, respiratory rate, urine output and condition of the wound till the patients got completely stabilized.

Appropriate care was given to burn wound in the form of antiseptic dressings or antiseptic application with exposure. Patients were observed daily for any clinical evidence of sepsis. Pus for culture and sensitivity was sent when required. Blood culture was sent whenever there was any clinical evidence of septicemia.

The results so obtained were analysed and compared so as to find out the relationship of platelet counts to burn wound sepsis and their efficacy in predicting morbidity and mortality in burn cases.

\section{Results}


Table 1: Mean Platelet Count in Survivors and Non-Survivors

\begin{tabular}{|l|l|l|}
\hline Day & Survivors & Non-survivors \\
\hline 1 & 2.59 & 2.01 \\
\hline 3 & 2.38 & 0.71 \\
\hline 7 & 2.28 & 0.22 \\
\hline 14 & 2.49 & 0.17 \\
\hline 21 & 2.61 & - \\
\hline
\end{tabular}

Mean platelet count on first day was 2.59 lacs in survivors whereas in case of non-survivors mean platelet count on first day was 2.01 lacs. On day 3, mean platelet count in survivors was 2.38 and in non-survivors it was only 0.71 . On 14th day, mean platelet count in survivors was 2.49 and in non-survivors it was 0.17 . On day 21 mean platelet count in survivors was 2.61 and in non-survivors it was 0 .

Table 2: Showing type of bacteria in blood culture

\begin{tabular}{|l|l|l|}
\hline Type of organism & No. of cases & \%age \\
\hline Staph Aureus & 6 & 24 \\
\hline E. Coli & 4 & 16 \\
\hline
\end{tabular}

This table shows type of bacteria in the blood culture. Six cases had growth of staph aureus in their blood culture while 16 cases E. Coli in their blood culture.

Table 3: Blood culture of burn patients in survivors and non-survivors

\begin{tabular}{|l|l|l|l|l|l|}
\hline \multirow{2}{*}{ Blood culture } & \multicolumn{3}{|l|}{ survivors } & \multicolumn{3}{|c|}{ Non-survivors } & \multirow{2}{*}{ Total } \\
\cline { 2 - 5 } & No. of cases & \%age & No. of cases & \%age & \\
\hline Positive & 4 & 16 & 6 & 24 & 10 \\
\hline Negative & 13 & 52 & 2 & 8 & 15 \\
\hline
\end{tabular}

Above table shows blood culture and survival of burn patients. Amongst survivors, only 4 patients had positive blood culture while $52 \%$ had no growth in their blood culture. Whereas in case of non-survivors $24 \%$ of the cases had positive blood culture and only $8 \%$ patients had no growth in the blood culture.

Table 4: Mean platelet count and blood culture in burn cases

\begin{tabular}{|l|l|l|l|}
\hline \multirow{2}{*}{$\begin{array}{l}\text { Platelet } \\
\text { Count }\end{array}$} & Blood culture & \multirow{2}{*}{ Total } \\
\cline { 2 - 3 } & Positive & Negative & \\
\hline Low $(<1.5)$ & 6 & 1 & 7 \\
\hline Normal & 4 & 14 & 18 \\
\hline Total & 10 & 15 & 25 \\
\hline
\end{tabular}

The mean platelet count and blood culture in burn cases. Six cases with low platelet count had positive blood culture while only 4 patients with normal platelet count had positive blood culture.

Table 5: Mortality

\begin{tabular}{|l|l|l|}
\hline Outcome & No. of patients & \% age \\
\hline Deaths & 8 & 32 \\
\hline Survived & 17 & 68 \\
\hline
\end{tabular}

Above table shows outcome of this study. Eight (32\%) of the total patients died and $68 \%$ survived.

\section{Discussion}

In the present study, mean platelet count decreased up to day 7 in both survivors and non-survivors. However from day 7 onwards, platelet counts steadily rose in case of survivors while maintaining a downward trend in case of non-survivors. Jefferson (2007) reported that $42.9 \%$ of nonsurvivors had $<100,000$ platelets and $92 \%$ of them died. Vasantharaja (2016) found that the fall in platelet count is associated with bad prognosis in burn patients [35].
In the present study staph aureus (6) was the dominant organism followed by E. coli (4) in blood culture. R Bagdonas (2003) concluded staph aureus as most common infection in burn patients. T Alebachew (2011) mentioned staph aureus as most common isolate in their study; similarly Vasantharaja (2016) also reported growth of staph aureus in 18 cases of their study.

In present study, only 4 patients had positive blood culture while $52 \%$ had no growth in their blood culture. Whereas in case of non-survivors $24 \%$ of the cases had positive blood culture and only $8 \%$ patients had no growth in the blood 
culture. The relationship between bacteremia and mortality was studied in 5882 burn patients consecutively admitted to one burn center between 1959 and 1983. Among 5877 patients with adequate data, 1481 had one or more positive blood cultures; 1529 patients died. A predictor of mortality was developed, based on data from the 4396 patients without positive blood cultures, and used to assign a discrete probability of death in the absence of bacteremia to all the patients. Comparisons were then made between observed and predicted mortality in subsets of patients with bacteremia.In 1169 inpatients admitted to the burn unit during the study period, $212(18 \%)$ had suspected sepsis, and $65(6 \%)$ had confirmed bacteremia. Sepsis was considered the primary cause of death in 198 patients $(65 \%$; $95 \%$ CI $65-70$ ) of the 304 patients that died.

\section{Conclusions}

To conclude, it may be said that the factors which contribute towards increased morbidity and mortality in burn cases are advanced age of the patient, extent and depth of burns and delay in starting treatment. However, the development of sepsis with its complications is the most important cause of morbidity and mortality.

Mortality was found to be significantly higher among those patients whose blood cultures were positive. Thrombocytopenia occurred in most of the cases with positive blood cultures indicating thereby that it is an important predictor of development of sepsis and outcome. Also thrombocytopenia preceded the development of early predictors of sepsis like temperature, loss of appetite, enteral feeding intolerance, tachycardia and leukocytosis. So it can be concluded that serial platelet counts can be independently used to identify those at risk to develop systemic infection, its complications and final outcome.

\section{Reference}

1. Abdolaziz, RastegarLari, Reza Alaghehbandan Nosocomial infections in an Iranian burn care center Burns Volume 26, Issue 8, December 2000, Pages $737-40$

2. Gajbhiye AS, Meshram MM, Kathod AP. Platelet count as a prognostic indicator in burn septicemia. Indian J Surg. 2013; 75(6):444-448. doi:10.1007/s12262-012-0532-6

3. Vostrugina K, Gudaviciene D, Vitkauskiene A. Bacteremias in patients with severe burn trauma. Medicina (Kaunas). 2006; 42(7):576-579.

4. Mason AD, McManus AT, Pruitt BA. Association of Burn Mortality and Bacteremia: A 25-Year Review. Arch Surg. 1986; 121(9):1027-1031. doi:10.1001/archsurg.1986.01400090057009

5. Jefferson L S, Joao B. predictive factors of mortality in burn patients. Med. Trop. S. Paulo.2007. 49 (6) 365-70

6. Vasantaraja T G, Narayan Moorthy R. Platelet count in predicting outcome in burn patient-A prospective study.IOSR-JDMS. 2016; p-ISSN 2279-0861. Vol 15(4)66-8

7. Bagdonas, R. \&Tamelis, A. \&Rimdeika, Rytis\&Kiudelis, Mindaugas. (2004). Analysis of burn patients and the isolated pathogens. Lithuanian Surg. 2. 190-3.

8. Alebachew T, Yismaw G, Derabe A, Sisay Z. Staphylococcus aureus burn wound infection among patients attending yekatit 12 hospital burn unit, addisababa, ethiopia. Ethiop J Health Sci. 2012; 22(3):209-213. 